\title{
Variable Clinical Expression in Patients with Mosaicism for KCNQ2 Mutations
}

\section{Mathieu Milh, ${ }^{1,2,3}$ Caroline Lacoste, ${ }^{1,2,4}$ Pierre Cacciagli, ${ }^{1,2,4}$ Affef Abidi, ${ }^{1,2}$ Julie Sutera-Sardo, ${ }^{1,2,3}$ Ilias Tzelepis, ${ }^{1,2}$ Estelle Colin, ${ }^{5}$ Catherine Badens, ${ }^{1,2,4}$ Alexandra Afenjar, ${ }^{6}$ Anne Dieux Coeslier, Thomas Dailland, ${ }^{8}$ Gaetan Lesca, ${ }^{9}$ Nicole Philip, ${ }^{1,2,4}$ and Laurent Villard ${ }^{1,2 *}$}

\author{
${ }^{1}$ Inserm, UMR S 910, Génétique Médicale et Génomique Fonctionnelle, Marseille, France \\ ${ }^{2}$ Aix Marseille Université, GMGF, Faculté de Médecine, Marseille, France \\ ${ }^{3}$ APHM, Hôpital d'Enfants de La Timone, Service de Neurologie Pédiatrique, Marseille, France \\ ${ }^{4}$ APHM, Hôpital d'Enfants de La Timone, Département de Génétique Médicale, Marseille, France \\ ${ }^{5}$ Département de Biochimie et Génétique, CHU d’Angers, Angers, France \\ ${ }^{6}$ APHP, Hôpital Trousseau, Service de génétique clinique et Service de neuropédiatrie, Paris, France \\ 'Clinique de Génétique, Hôpital Jeanne de Flandre, Lille, France \\ ${ }^{8}$ Centre Hospitalier des Pays de Morlaix, Pédiatrie et Néonatalogie, Morlaix, France \\ ${ }^{9}$ Département de Génétique Médicale, Hospices Civils de Lyon, Université Lyon 1, Lyon, France
}

\begin{abstract}
Mutations in the $K C N Q 2$ gene, encoding a potassium channel subunit, were reported in patients presenting epileptic phenotypes of varying severity. Patients affected by benign familial neonatal epilepsy (BFNE) are at the milder end of the spectrum, they are affected by early onset epilepsy but their subsequent neurological development is usually normal. Mutations causing BFNE are often inherited from affected parents. Early infantile epileptic encephalopathy type 7 (EIEE7) is at the other end of the severity spectrum and, although EIEE7 patients have early onset epilepsy too, their neurological development is impaired and they will present motor and intellectual deficiency. EIEE7 mutations occur de novo. Electrophysiological experiments suggested a correlation between the type of mutation and the severity of the disease but intra and interfamilial heterogeneity exist. Here, we describe the identification of KCNQ2 mutation carriers who had children affected with a severe epileptic phenotype, and found that these individuals were mosaic for the KCNQ2 mutation. These findings have important consequences for genetic counseling and indicate that neurological development can be normal in the presence of somatic mosaicism for a KCNQ2 mutation.
\end{abstract}

\section{INTRODUCTION}

KCNQ genes encode voltage-gated potassium channel subunits. Functional KCNQ channels are composed of homomeric or heteromeric assemblies of 4 subunits. In neuronal cells, KCNQ2 and KCNQ3 heterotetramerize to give rise to the $\mathrm{M}$ current $\left(\mathrm{I}_{\mathrm{M}}\right)$, a key player for the regulation of neuronal excitability [Brown and Passmore, 2009]. Until recently, KCNQ2 (OMIM 602235) and KCNQ3 (OMIM 602232) mutations had only been reported in benign familial neonatal epilepsy (BFNE, OMIM 121200) [Biervert et al., 1998; Singh et al., 1998] and peripheral nerve hyper excitability or myokymia [Dedek et al., 2001]. Patients suffering from BFNE present stormy partial motor seizures starting few days after birth, normal or subnormal interictal EEG activity and usually normal cognitive development. The epilepsy usually stops before the end of 
the 3rd month. In BFNE, KCNQ2 mutations are transmitted from affected individual to affected individual following a classical autosomal dominant inheritance mode. More recently, mutations in the $K C N Q 2$ gene were also described in a very severe epileptic phenotype called early infantile epileptic encephalopathy (EIEE) subtype 7 (OMIM 613720) [Dedek et al., 2003; Weckhuysen et al., 2012]. Here, contrary to what is observed in BFNE, the patients present a severe phenotype with most often a suppression burst EEG pattern and frequent polymorphic seizures. In most cases, the evolution is poor with severe neurological impairment accompanied by severe intellectual deficiency. Furthermore, a proportion of patients die within the first years of life. Mutations causing EIEE7 had occurred de novo in most cases [Weckhuysen et al., 2012]. In most cases, the mutations causing BFNE alter a different amino acid than the mutations causing EIEE7, revealing a significant genotype-phenotype correlation for these two epileptic disorders. Given that mutations causing EIEE7 are usually found to have occurred de novo, a variant in the KCNQ2 gene inherited from a healthy parent would likely be considered to be a non-pathogenic variant. However, we show here that variants in the KCNQ2 gene can be inherited from an apparently healthy parent and yet cause a severe phenotype, a discovery that has strong implications for pediatric neurologists, clinical geneticists, and genetic counselors.

\section{MATERIALS AND METHODS Cohort and DNA Samples}

The samples used in this study come from a research cohort assembled during the course of a clinical research project (PHRC IR 2011, Marseille University Hospital) and from the diagnostic activity of the departments of Medical Genetics of La Timone Children's Hospital, Marseille and Lyon University Hospital, Lyon, France. Inclusion criteria for the patients were the presence of recurrent seizures occurring before 1 month of age in a patient with a normal metabolic profile and normal MRI. This project was approved by the local ethics committee (Comite de Protection des Personnes Sud Méditerranée II). DNA samples were prepared using standard procedures from blood, and from buccal cells or hair roots when possible.

\section{Sequencing, Cloning and Mosaicism Analysis}

Primers used to amplify KCNQ2 exons were designed starting from reference sequence NM_172107. All exons were amplified using total genomic DNA (primer sequences are available upon request) and sequenced on a 3130XL automated sequencer (Thermo Fisher Inc., Waltham, MA). For carriers where alternative DNA sources were available, the PCR amplicons containing the mutation were subcloned in pGEM-T and transformed into XL1 Blue cells (Agilent Technologies Inc., Santa Clara, CA). One hundred clones for each transformation were sequenced using the Big Dye kit (Thermo Fisher).

\section{RESULTS}

We used Sanger sequencing to screen the coding exons of KCNQ2 in DNA samples from patients suffering from epileptic encepha- lopathy. All patients had intellectual disability and epilepsy that started before 1 month of age. EEG data could not be collected for all cases. In that cohort, we identified 43 variants in the KCNQ2 gene in 208 patients $(20.6 \%)$ (Table I). Among these, 21 variants had arisen de novo, 14 variants were found to be inherited from an unaffected parent and for the remaining eight variants, unavailability of parental DNA did not allow us to determine if the mutation was inherited or de novo. Among the variants inherited from unaffected parents, we identified the c.881C $>$ T/p.A294V mutation in a child (patient 1) with a severe phenotype, with seizure onset in the first days of life and a suppression burst EEG pattern. Patient 1 was initially diagnosed as having Ohtahara syndrome. She became seizure-free within the first year of life, with global developmental delay. Interestingly, the p.A294V mutation is a hotspot mutation in KCNQ2 that has been identified five times in our diagnostic cohorts and twice in the literature [Steinlein et al., 2007; Kato et al., 2013]. All reported patients had de novo occurrences and all suffered from a severe phenotype. To our surprise, the p.A294V mutation, identified in patient 1 was also found in his unaffected mother (data not shown). We showed that this mutation alters the $\mathrm{I}_{\mathrm{M}}$ current and the localization of the KCNQ2/KCNQ3 tetramers within neurons [Abidi et al., 2015]. The recurrence of the p.A294V mutation in patients with a similar phenotype and its impact on the function of the KCNQ channels made it very unlikely to be a benign variant. We thus amplified a DNA segment containing the p.A294V mutation using DNA prepared from buccal swabs, hair root, and the blood of the carrier mother to study tissues of different embryonic origin. PCR products were prepared using two different PCR reactions and were cloned in pGEM-T. For each tissue, $2 \times 50$ clones were sequenced and the clones harboring the $\mathrm{c} .881 \mathrm{C}>\mathrm{T}$ mutation were counted. The results of this analysis showed that the patient's mother carried the mutation in $30 \%$ of clones in the PCR product originating from blood, whereas her affected daughter has $49 \%$ of mutant clones in similar conditions, as expected for a heterozygous carrier. The percentage of mutant clones was $29 \%$ and $51 \%$ in the DNA prepared from buccal cells and hair roots, respectively. These results indicate that the mother of Patient 1 is a mosaic carrier for the c. $881 \mathrm{C}>\mathrm{T}$ mutation. We went back to the available medical files of the mother and found that she had experienced a few neonatal seizures, diagnosed as benign neonatal epilepsy. She had been free of seizures since then. She developed normally, with good social integration but with low educational achievements.

Based on these findings, we retrospectively analyzed the medical files of all the apparently healthy parents who had transmitted a KCNQ2 variant to a severely affected child (see Table I). In our series, we had eight maternal and six paternal transmissions in 14 patients (including the p.A294V mutation described above). All the variants were different and seven of them were synonymous substitutions considered to be polymorphisms although absent from dbSNP build 141. For the remaining six missense mutations that had been inherited from a healthy parent (p.A253T, p.P420M, p.V422I, p.A501P, p.M551T, and p.R871S), examination of the Sanger sequencing traces revealed similar peak intensities between the affected child and the carrier parent. However, the mother of Patient 2, who was a carrier for the p.A253T mutation, had experienced neonatal seizures that lasted several days. She 
TABLE I. Summary of the Variants Identified in the KCNQ2 Gene in 208 E0EE Patients. Mutations are Numbered Based on the Reference Sequence NM_172107. All Variants Were Confirmed Using a Second Sample. Parental Identity was Confirmed Using a set of 5 Highly Polymorphic Microsatellites. The Inheritance Pattern and SNP Referenced in dbSNP Build 141 at the

Same Amino Acid Position are Provided

\begin{tabular}{l} 
Protein variation \\
p.M1V \\
p.V175L \\
p.G189V \\
p.L203P \\
p.R2070 \\
p.R207W \\
p.R213W \\
p.G239R \\
p.A253T \\
p.A265V \\
p.A265V \\
p.T274M \\
p.T274M \\
p.G279C \\
p.T287N \\
p.G290S \\
p.A294V \\
p.A294V \\
p.A294V \\
p.A294V \\
p.A294V \\
p.A294V \\
p.G301S \\
p.F304S \\
p.A306V \\
p.A309V \\
Splicing defect \\
p.L351L \\
p.R353H \\
p.L356V \\
p.S401S \\
p.P420M \\
p.V422I \\
p.A501P \\
p.P534P \\
p.R541G \\
p.R547R \\
p.M551T \\
p.D563N \\
p.D563D \\
p.P652P \\
p.C774Lf*91 \\
p.R871S \\
\\
\hline
\end{tabular}

\section{Nucleotide variation}

c. $1 \mathrm{~A}>\mathrm{G}$

c. $523 G>T$

c. $566 \mathrm{G}>\mathrm{T}$

c.608T $>C$

c. $620 \mathrm{G}>\mathrm{A}$

c.619C $>\mathrm{T}$

c. $637 \mathrm{C}>\mathrm{T}$

c. $715 G>C$

c. $757 \mathrm{G}>\mathrm{A}$

c.794C $>\mathrm{T}$

c. $794 C>T$

c. $821 \mathrm{C}>\mathrm{T}$

c. $821 \mathrm{C}>\mathrm{T}$

c. $835 \mathrm{G}>\mathrm{T}$

c. $860 \mathrm{C}>\mathrm{A}$

c. $868 \mathrm{G}>\mathrm{A}$

c. $881 \mathrm{C}>\mathrm{T}$

c. $881 \mathrm{C}>\mathrm{T}$

c. $881 \mathrm{C}>\mathrm{T}$

c. $881 \mathrm{C}>\mathrm{T}$

c. $881 \mathrm{C}>\mathrm{T}$

c. $881 \mathrm{C}>\mathrm{T}$

c. $901 \mathrm{G}>\mathrm{A}$

c. $911 \mathrm{~T}>\mathrm{C}$

c. $917 \mathrm{C}>\mathrm{T}$

c. $926 C>T$

c. $1024-2 A>G$

c. $1053 C>T$

c. $1058 \mathrm{G}>\mathrm{A}$

c. $1066 C>G$

c. $1203 \mathrm{~T}>\mathrm{C}$

c. $1259 C>T$

c. $1264 \mathrm{G}>\mathrm{A}$

c. $1501 G>C$

c. $1602 G>A$

c. $1621 A>G$

c. $1644 G>A$

c. $1652 \mathrm{~T}>\mathrm{C}$

c. $1687 \mathrm{G}>\mathrm{A}$

c. $1689 C>T$

c. $1956 \mathrm{G}>\mathrm{A}$

c.2318insG

c. $2613 G>T$

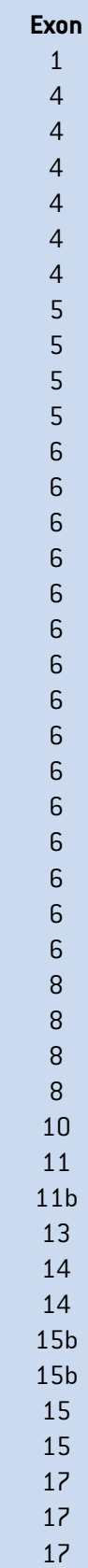

Inheritance
Unknown
De novo
Unknown
De novo
De novo
Unknown
De novo
De novo
Maternal
De novo
Unknown
De novo
De novo
Unknown
De novo
De novo
De novo
De novo
De novo
De novo
Paternal
Maternal
De novo
De novo
Unknown
De novo
Unknown
Maternal
De novo
Unknown
Paternal
Paternal
Paternal
Maternal
Maternal
De novo
Maternal
Paternal
De novo
Maternal

dbSNP build 141 p.M1V ${ }^{a}$, p.M1T
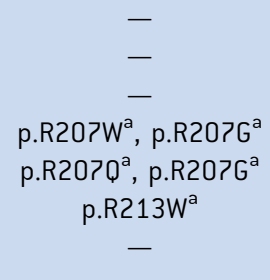

p.A265A

p.A265A

p.T274T

p.T274T

-

p.T287T

p.G290D

p.A294G ${ }^{a}$

p.A294G ${ }^{a}$

p.A294G ${ }^{a}$

p.A294G ${ }^{a}$

p.A294G ${ }^{a}$

p.A294G ${ }^{a}$

p.F304F

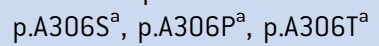

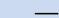

$-$

p.R353G ${ }^{\mathrm{a}}$

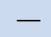

p.P420L ${ }^{a}$

$-$

p.A501A
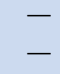

$-$

p.D563E

p.D563E

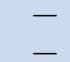

p.R87 $1 G^{a}$

${ }^{a}$ Denotes Predicted Pathogenicity According to dbSNP 141.

developed normally with a normal intellectual state. Her daughter was born at term. She experienced tonic asymmetric seizures in a stormy way beginning during the first day of life. She was admitted in intensive neonatal care unit. Interictal EEG showed multifocal spikes, without suppression-burst. She rapidly became seizure-free with a normal EEG. At 3 years of age, she has a global develop- mental delay with absence of language. Unfortunately, despite several attempts, we were not able to obtain new samples or samples originating from different tissues for the carrier mother of Patient 2 to study putative somatic mosaicism in different tissues.

In the cohort of patients from Lyon, we found a mosaic state in two parents who had transmitted their respective mutation to a 
child with EIEE. Patient 3 experienced tonic and tonic-clonic seizures at day 1. EEG showed slow background activity with bilateral paroxysmal discharges. Seizures were controlled at 3 weeks of age by valproate. At 16 months of age, she had slight motor and speech delay and stereotypic movements of the hands. Brain MRI at 1 month of life was normal. Sanger sequencing showed the presence of the p.A294V mutation, in a $20 \%$ mosaic state in the father. $\mathrm{He}$ had experienced seizures from the neonatal period that persisted until childhood. This patient is now seizure-free and has normal cognitive development. The second patient from Lyon was born at 31 weeks of pregnancy. She first experienced focal seizures at 1.5 months of age, corresponding to term in corrected age. She had frequent focal tonic seizures and clusters of spasms. EEG showed multifocal spikes. Epilepsy was initially resistant to valproate and was controlled with vigabatrin, and levetiracetam. Brain MRI was normal. At last examination, she was aged two. She has been free of seizures since the age of 6 months with levetiracetam. EEG was normal. Psychomotor development was mildly delayed. Genetic analysis found the c.997C $>$ T/p.R333W mutation. This mutation was also found in low mosaic state $(5 \%)$ by Sanger sequencing in the mother, who had no history of neonatal seizures. Taken together, we found three confirmed mosaic carriers of a KCNQ2 mutation and one possible carrier. Three of them were affected by neonatal epilepsy, but with subsequently normal neurological development.

\section{DISCUSSION}

Our results demonstrate that a KCNQ2 mutation can be inherited from an apparently healthy parent and yet cause a severe neurological phenotype in the affected child. A literature survey revealed that two previously published KCNQ2 mutations were proposed to be mosaic. The first mutation was a c.854C $>$ A/p.P285H mutation in a patient with moderate intellectual disability at 2 years of age [Kato et al., 2013] that was also found in her carrier mother, in whom Sanger sequencing performed in 46 clones showed $28 \%$ of mosaicism (13/46 clones were carrying the variant). The second mutation was a c.638G $>$ A/p.R213Q substitution but it was inherited from a symptomatic carrier father, in whom the level of mosaicism was estimated to be $30 \%$ as shown by restriction digest of PCR fragments. The carrier father had myokymia, epilepsy between 4 days to 11 weeks and he is still on carbamazepine therapy at age 32 [Weckhuysen et al., 2012]. The same amino acid was affected by the c.637C $>$ T/p.R213W mutation found to have occurred de novo in a severely affected individual in our cohort but also in a family presenting BFNE [Sadewa et al., 2008]. Analysis of the electrophysiological properties of the two mutations suggests that the p.R213W mutation has a milder effect on the function of the potassium channel than the p.R213Q mutation [Miceli et al., 2008]. The presence of that same mutation in a severely affected individual in our cohort is currently unexplained, although intra and interfamilial variability was previously documented for KCNQ2 mutations [Soldovieri et al., 2014].

In our study, the level of mosaicism detected in blood samples ranges from $5 \%$ to $30 \%$ of cells. The neurological development of the mosaic carriers was normal in all cases, suggesting that up to $30 \%$ of cells carrying a mutation in KCNQ2 is not sufficient to impair neurological development. In contrast, it seems that a low rate of mutated neurons is sufficient to cause neonatal seizures. Importantly, the transmission of these mutations to severely affected children indicates that pediatric neurologists, clinical geneticists, and genetic counselors need to consider mosaicism for all inherited variants in the KCNQ2 gene. Conversely, our results raise new questions in genetic counseling of patients presenting with BFNE. Genetic counseling is easy in case of a familial transmission with all affected individuals presenting the same benign course of the disease. However, when a de novo KCNQ2 mutation is identified in an individual presenting with $\mathrm{BFNE}$, or when family investigation is not possible, careful attention must be paid to the detection of somatic mosaicism. Such mosaic individuals are potentially at risk of giving birth to children suffering from EIEE7.

In conclusion, we recommend that all inherited mutations in KCNQ2 be checked for: (1) the presence of subtle epileptic phenotypes in early infancy in the carrier parent; (2) the uneven distribution of the two alleles on Sanger sequencing results (or other mutation screening techniques). Finally, these findings also suggest that in order to cause a neurological disease, KCNQ2 mutations causing EIEE7 must be present in a sufficient proportion of cells and that the neurological development can proceed normally after birth with up to $30 \%$ of abnormal KCNQ2 subunits.

\section{ACKNOWLEDGMENTS}

The authors thank all patients and their families for their participation in this study. This work was supported by Programme Hospitalier de Recherche Clinique 2011, Inserm and Aix Marseille University. We thank the Centre de Ressources Biologiques of La Timone Children's Hospital (Karine Bertaux, Cécile Mouradian and Andrée Robaglia-Schlupp) for assistance with the samples used in this study and Raphaelle Lamy for expert technical assistance.

\section{REFERENCES}

Abidi A, Devaux JJ, Molinari F, Alcaraz G, Michon FX, Sutera-Sardo J, Becq H, Lacoste C, Altuzzara C, Afenjar A, Mignot C, Doummar D, Isidor B, NGuyen S, Colin E, De la Vaissière S, Haye D, Trauffler A, Badens C, Prieur F, Lesca G, Villard L, Milh M, Aniksztejn L. 2015. Functional and subcellular consequences of a recurrent KCNQ2 mutation associated to early epileptic encephalopathy: A moderate effect on $\mathrm{M}$ current but a strong impact on Kv7 channels neuronal targeting. Neurobiol Dis, in press.

Biervert C, Schroeder BC, Kubisch C, Berkovic SF, Propping P, Jentsch TJ, Steinlein OK. 1998. A potassium channel mutation in neonatal human epilepsy. Science 279:403-406.

Brown DA, Passmore GM. 2009. Neural KCNQ (Kv7) channels. Br J Pharmacol 156:1185-1195.

Dedek K, Kunath B, Kananura C, Reuner U, Jentsch TJ, Steinlein OK. 2001. Myokymia and neonatal epilepsy caused by a mutation in the voltage sensor of the KCNQ2 K+ channel. Proc Natl Acad Sci USA 98:12272-12277.

Dedek K, Fusco L, Teloy N, Steinlein OK. 2003. Neonatal convulsions and epileptic encephalopathy in an italian family with a missense mutation in the fifth transmembrane region of KCNQ2. Epilepsy Res 54:21-27.

Kato M, Yamagata T, Kubota M, Arai H, Yamashita S, Nakagawa T, Fujii T, Sugai K, Imai K, Uster T, Chitayat D, Weiss S, Kashii H, Kusano R, 
Matsumoto A, Nakamura K, Oyazato Y, Maeno M, Nishiyama K, Kodera H, Nakashima M, Tsurusaki Y, Miyake N, Saito K, Hayasaka K, Matsumoto N, Saitsu H. 2013. Clinical spectrum of early onset epileptic encephalopathies caused by KCNQ2 mutation. Epilepsia 54:12821287.

Miceli F, Soldovieri MV, Ambrosino P, Barrese V, Migliore M, Cilio MR, Taglialatela M. 2008. Genotype-phenotype correlations in neonatal epilepsies caused by mutations in the voltage sensor of $\mathrm{K}(\mathrm{v}) 7.2$ potassium channel subunits. Proc Natl Acad Sci USA 110:43864391.

Sadewa AH, Sasongko TH, Gunadi, Lee MJ, Daikoku K, Yamamoto A, Yamasaki T, Tanaka S, Matsuo M, Nishio H. 2008. Germ-line mutation of KCNQ2, p.R213W, in a Japanese family with benign familial neonatal convulsion. Pediatr Int 50:167-171.

Singh NA, Charlier C, Stauffer D, DuPont BR, Leach RJ, Melis R, Ronen GM, Bjerre I, Quattlebaum T, Murphy JV, McHarg ML, Gagnon D, Rosales TO, Peiffer A, Anderson VE, Leppert M. 1998. A novel potassium channel gene, KCNQ2, is mutated in an inherited epilepsy of newborns. Nat Genet 18:25-29.

Soldovieri MV, Boutry-Kryza N, Milh M, Doummar D, Heron B, Bourel E, Ambrosino P, Miceli F, De Maria M, Dorison N, Auvin S, Echenne B, Oertel J, Riquet A, Lambert L, Gerard M, Roubergue A, Calender A, Mignot C, Taglialatela M, Lesca G. 2014. Novel KCNQ2 and KCNQ3 mutations in a large cohort of families with benign neonatal epilepsy: first evidence for an altered channel regulation by syntaxin-1A. Hum Mutat 35:356-367.

Steinlein OK, Conrad C, Weidner B. 2007. Benign familial neonatal convulsions: Always benign? Epilepsy Res 73:245-249.

Weckhuysen S, Mandelstam S, Suls A, Audenaert D, Deconinck T, Claes LR, Deprez L, Smets K, Hristova D, Yordanova I, Jordanova A, Ceulemans B, Jansen A, Hasaerts D, Roelens F, Lagae L, Yendle S, Stanley T, Heron SE, Mulley JC, Berkovic SF, Scheffer IE, de Jonghe P. 2012. KCNQ2 encephalopathy: Emerging phenotype of a neonatal epileptic encephalopathy. Ann Neurol 71:15-25. 